\title{
Neglected Thoraco Lumbar Traumatic Spine Injuries
}

\author{
Kavin Khatri ${ }^{1}$, Kamran Farooque ${ }^{2}$, Vijay Sharma ${ }^{2}$, Babita Gupta ${ }^{3}$, Shivanand Gamanagatti ${ }^{4}$ \\ ${ }^{I}$ Department of Orthopaedics, GGS Medical College, Faridkot, India \\ ${ }^{2}$ Department of Orthopaedics, All India Institute of Medical Sciences, New Delhi, India \\ ${ }^{3}$ Department of Anaesthesia, All India Institute of Medical Sciences, New Delhi, India \\ ${ }^{4}$ Department of Radiology, All India Institute of Medical Sciences, New Delhi, India
}

\section{Study Design: Retrospective study.}

Purpose: To outline the etiology, complications and management difficulties encountered in the management of neglected thoracolumbar spine injuries.

Overview of Literature: The English literature describes overlooked diagnosis as the most common cause of neglected spine injuries. However, the reasons differ in developing or under-developed nations. Moreover, there is scarcity of literature about the neglected spinal injuries.

Methods: Patients presenting with thoracolumbar traumatic injuries who had not received any form of treatment for more than three weeks were included in the study. The demographic details, operative procedure performed and complications encountered, along with American Spinal Injury Association grade and spinal cord independence measure score recorded on the history sheets were noted. The data were analyzed.

Results: Forty patients were included in the study. Inadequate treatment at the first contact hospital (45\%) followed by late presentation (38\%) and missed injury (17\%) were the major etiological factors for the neglected traumatic injuries in the thoracolumbar spine. The most common complications seen in the management of these cases were pressure sores $(58 \%)$, back pain $(57 \%)$, urinary tract infection (42\%) and residual kyphotic deformity (42\%).

Conclusions: Management of neglected thoracolumbar injuries is challenging. The delay in presentation should not prevent spine surgeon in proceeding with operative intervention as good results can be expected.

Keywords: Thoracolumbar spine; Cord injury; Fracture; Spine; Trauma; Spinal cord injuries; Spinal fractures; Neglected diseases

\section{Introduction}

In developing countries like India, the prevalence of neglected traumatic thoracolumbar injuries is reportedly as high as $26.6 \%$ in few high volume centers catering to a large population [1]. The delay in presentation of thoracolumbar injuries has an impact on its management and rehabilitation. Cases presenting after three weeks of injury may require different management strategies than cases presenting at an early stage [2]. Optimal treatment remains controversial due to the paucity of literature on neglected traumatic thoracolumbar spinal injuries $[1,3]$. Overlooked diagnosis has been implicated as the major reason for neglect in these patients in western literature. However, the same may not be true in case of developing nations like India.

Received Nov 16, 2015; Revised Jan 17, 2016; Accepted Jan 17, 2016

Corresponding author: Kamran Farooque

Department of Orthopaedics, All India Institute of Medical Sciences,

Ansari Nagar (west), Ring road, New Delhi, India. Pin code-110069

Tel: +98-1122-9136, E-mail: kamran.farooque@gmail.com 
The purpose of this study was to determine the cause, complications and treatment challenges in the management of neglected thoracolumbar spine injuries in underdeveloped or developing countries. We report our experience with 40 cases of neglected traumatic thoracolumbar spinal injuries treated at our level one trauma center over the last seven years.

\section{Materials and Methods}

The study was conducted with the approval of the ethics committee of the institution (Institute where study was carried out: All India Institute of Medical Sciences, Ansari Nagar (west), New Delhi, India). Inpatient records of patients diagnosed with thoracolumbar traumatic injuries of more than three weeks duration between January 2008 to March 2014 were searched. Patients with cervical spine injury were excluded. Selected patients were contacted by telephone or letter, and were asked for an additional visit to our hospital. All patients provided informed consent to participate in this study. Forty patients were willing to participate. Another 32 who met the inclusion criteria were not willing to participate, did not respond to our invitation to participate or could not be traced. Patients who had received surgical management before presentation to the institute were excluded. Patients (33 males, seven females) ranged in age from 9 to 58 years with a mean age of 25.95 years. The injuries were sustained in falls from a height $(n=37)$, diving $(n=1)$ and traffic accident $(n=9)$.

The hospital records included clinical history sheet and operative notes. They were studied to determine the demographic data, mode of injury, duration and cause of delay, treatment given, complications associated with injury and treatment, preoperative and postoperative neurological status using American Spinal Injury Association (ASIA) grading along with spinal cord independence measure (SCIM) score. Preoperative and postoperative radiographs were analyzed to classify the injury and treatment.

The standard posterior midline approach to the spine was used in the majority of the cases. Anterior approach along with posterior approach was used in combination or in isolation in selected cases. Pedicle screw rod constructs (Medtronic, Minneapolis, MN, USA) were used for fixation and addition of mesh/expandable cage was left to the discretion of the treating surgeon. Postoperatively, the patients were mobilized in bed as per pain tolerance. Subse- quently the patients were mobilized in wheelchair or with support as peroperative the postoperative neurological status. SCIM score and ASIA grading was also recorded at the time of discharge and last follow up.

The functional assessment of the patients was done with SCIM [4]. It assesses the ability to perform routine daily activities in spinal cord injury patients and can be used to measure the outcome following spinal interventions. It includes 19 tasks grouped into four areas of function: self-care, respiration and sphincter management, mobility in room and toilet and indoor/outdoor mobility. Scoring was done by observation while a patient performed an assigned task. The most compatible score was entered in the SCIM evaluation sheet.

The end point was findings noted at the last follow-up. Data are presented as mean \pm standard deviation. Data was analyzed using analysis of variance with repeated measures for parametric data and Friedman test for nonparametric data. The statistical significance was defined at the $5 \%$ level $(p \leq 0.05)$.

\section{Results}

Forty patients were available for analysis in the present retrospective cohort study. Inadequate primary treatment at the first contact hospital (45\% followed by late presentation (38\%) and missed injury (17\%) were the major reasons for the neglected traumatic injuries in thoracolumbar spine (Table 1). Missed injury cases were the patients with associated head injury or polytrauma. In these cases the diagnosis was delayed due to presence of other injuries. The delay in definitive treatment was three to six weeks in 22 patients, between six to 12 weeks in 10 patients and more than 12 weeks in eight patients. The majority of the injuries were classified as burst fractures in 20 cases followed by fracture dislocations in 17 cases. There was single case each of traumatic spondylolisthesis, spondyloptosis and soft tissue chance fracture.

Anterior surgery alone was done in nine patients; posterior surgery in 26 cases and a combined anterior and posterior approach was required in five cases (Fig. 1). The most common complication encountered in the management of these cases was pressure sores, which was present in $58 \%$ of cases. Urinary tract infection, respiratory tract infection, and gastrointestinal complications were present in a substantial number of cases (Table 2). The ASIA grading recorded at presentation, discharge and at last 
Table 1. Reasons for delay in operative intervention in cases with neglected thoracolumbar injuries

\begin{tabular}{lc} 
Cause & Percentage of cases \\
Inadequate treatment at first contact & 32 \\
\hline a. Lack of adequate infrastructure and trained manpower & 8 \\
\hline b. Poor rehabilitation subsequent to initial treatment & 5 \\
\hline c. Ignorance among patients and physicians about the seriousness of the spinal injuries & 20 \\
\hline Is late presentation to hospital & 10 \\
\hline a. Non availability or complete absence of health care facilities & 8 \\
\hline b. Opting for other forms of treatment than modern allopathic medicine & 17 \\
\hline c. Lack of financial resources & 8 \\
\hline Missed injury due to head injury or polytrauma & \\
\hline
\end{tabular}
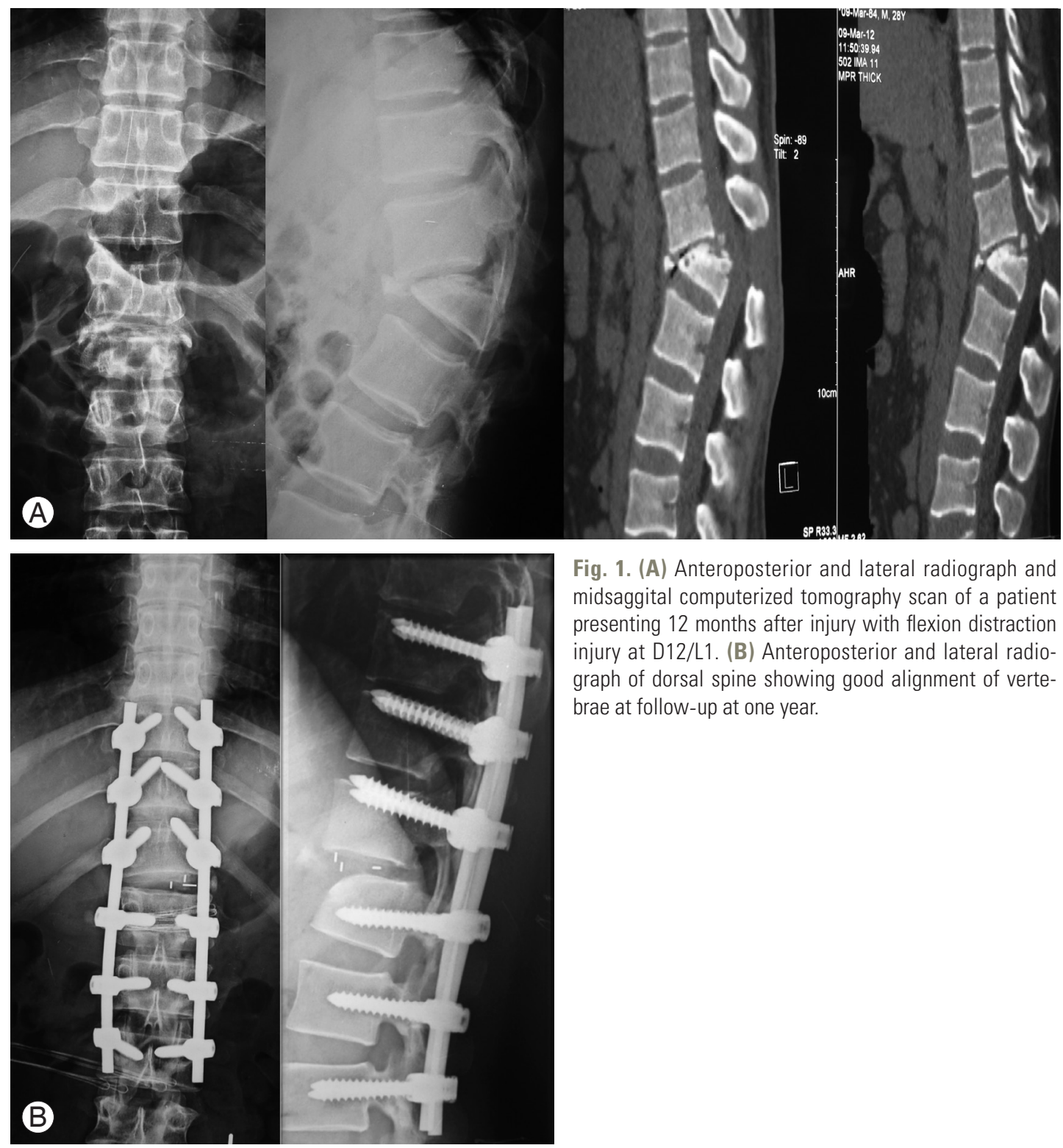

Fig. 1. (A) Anteroposterior and lateral radiograph and midsaggital computerized tomography scan of a patient presenting 12 months after injury with flexion distraction injury at D12/L1. (B) Anteroposterior and lateral radiograph of dorsal spine showing good alignment of vertebrae at follow-up at one year. 
follow-up along with SCIM are summarized in Table 3. No neurologic improvement was evident in 35\% $(n=14)$ patients. All were ASIA 'A' at the initial presentation. A neurologic improvement of at least one ASIA grade was seen in $52.5 \%(n=21)$ patients subsequent to operative intervention. Five patients (12.5\%) were ASIA 'E' at presentation and remained the same at follow-up. The average SCIM score at final follow up was 73. Average SCIM score was also calculated for individual spinal segment and improvement hence recorded was also subjected to test of significance (Table 4).

The patients were divided into three groups depend-

Table 2. Complications noticed in neglected spinal injuries

\begin{tabular}{lc} 
Complication & Percentage of cases \\
\hline Bed sore & 58 \\
\hline Back pain & 57 \\
Constipation & 54 \\
\hline Residual kyphotic deformity & 42 \\
\hline urinary tract infection & 42 \\
\hline Depression & 38 \\
\hline Deep vein thrombosis & 26 \\
\hline Joint contractures & 22 \\
\hline Paralytic ileus & 14 \\
\hline Respiratory tract infection & 10 \\
Calculi formation in kidney or bladder & 3 \\
\hline
\end{tabular}

ing upon the delay in definitive treatment of duration between three to six weeks, six to 12 weeks and more than 12 weeks. There was no statistically significant correlation found between delay in surgery and SCIM scores $(\mathrm{F}=2.140$, $p=0.13)$. The patients reported statistically significant improvement in SCIM scores at the time of the last followup. Improvement was irrespective of the level of vertebral injury (Table 3 ). The improvement in ASIA grade in patients presenting with ASIA 'A' was not statistically significant $(p=0.66)$. However, the improvement was significant after operative intervention in cases with higher grades of ASIA at initial contact $(p<0.05)$.

\section{Discussion}

The time frame to label a spinal injury as neglected is debatable, with reports describing cut-off times after the initial traumatic event of three weeks (patients limited to old distractive flexion injuries of the subaxial cervical spine) [5], three weeks for neglected spine dislocation [6], four weeks in a retrospective analysis of neglected spinal cord trauma [1], and more broadly as treatment that is not timely when treatment options are limited [3]. We used three weeks as the cut off time limit for labeling a spinal injury as neglected because in majority of the cases that is when fibrosis sets in around the injured segment and makes operative intervention difficult [2].

The most common cause for neglected thoracolumbar

Table 3. ASIA grade in patients at the time of admission, discharge and at the time of last follow-up

ASIA grade No. of patients at admission No. of patients at discharge No. of patients at final follow-up

\begin{tabular}{lrrr} 
A & 17 & 16 & 14 \\
B & 3 & 4 & 1 \\
C & 10 & 8 & 2 \\
D & 5 & 6 & 6 \\
E & 5 & 6 & 17 \\
\hline
\end{tabular}

ASIA, American Spinal Injury Association.

Table 4. Average SCIM score with respect to the vertebral segment of the injury at the time of admission, discharge and at the time of last follow-up

\begin{tabular}{lcccc} 
Vertebral segment & SCIM (admission) & SCIM (discharge) & SCIM (final follow-up) & F-value, $p$-value \\
Dorsal & 12.375 & 39.87 & 53.12 & $12.14,<0.05$ \\
$\begin{array}{l}\text { Dorsolumbar junction } \\
\text { (D12-L1) }\end{array}$ & 16.16 & 59.25 & 75.58 & $63.75,<0.05$ \\
Lumbar & 16.16 & 59.25 & 75.58 & $23.36,<0.05$ \\
\hline
\end{tabular}

SCIM, spinal cord independence measure. 
injuries was inadequate management at the initial hospital contact following injury. The reasons may be lack of adequate infrastructure as well as trained spine surgeons, and virtually nonexistent rehabilitation services. The patients were discharged early from our hospital without co-ordination with the available limited rehabilitation services due to high volume of orthopedic trauma patients being treated. Moreover, there is a lack of awareness regarding the rehabilitation services among spine surgeons in this part of the world. Lack of financial support for operative treatment and subsequent rehabilitation services were also major contributing reasons. Most patients with low socioeconomic status are not covered under any health insurance scheme. This was in contrast to the missed diagnosis as the major cause described in western literature. Poonnoose et al. [7] reported the incidence of missed injuries at $36 \%$ in thoracolumbar spine while Meldon and Moettus [8] reported a lower rate of $19.5 \%$. The cases of missed injuries could be prevented by adhering to protocols and hence avoid the late complications $[9,10]$. Krueger et al. [11] reported an incidence of missed injuries as $11 \%$ and advised computed tomography in cases of isolated lumbar transverse process fracture to avoid missing an occult spinal injury. Other studies have advised imaging of the whole spine to look for noncontiguous lesions $[12,13]$.

Health care facilities were not accessible in $20 \%$ of our cases. This problem is faced in many developing nations globally. Few hospitals in our country cater to large population leading to long waiting period and poor rehabilitation.

In $10 \%$ of our cases an alternate form of treatment include homeopathic, Unani and Ayurvedic medicine was tried first, with definitive modern allopathic care being delayed.

Although the incidence of missed injury in thoracolumbar region is lower than that of cervical region, secondary neurological deterioration is observed more commonly in thoraco lumbar spine mainly due to the narrow spinal canal in the region [14]. These injuries can also present with persistent pain and progression of deformity along with secondary neurological deterioration.

The surgical management of old neglected spine injuries is difficult, lengthy and fraught with potential neurological complications. The injuries, which could have been managed without any operative intervention, may require surgery to treat the late complications, especially in cases of missed spine injuries due to delayed presentation of ky- photic deformity [3].

Neglected thoracolumbar spine injuries may require extensive anterior procedures more frequently than isolated posterior indirect reduction maneuvers to achieve correct alignment. In our study, the anterior approach was used in $22 \%$ of cases. Bohlman [15] had also advocated anterior decompression in cases of old painful and paralytic patients of thoracolumbar injuries. In few cases, a combined anterior and posterior approach may be required to correct progressive kyphotic deformity [16]. There is delayed rehabilitation following these extensive procedures and the results achieved are also suboptimal. In many cases even the restoration of normal spinal alignment is not aimed for, due to long standing intervertebral fusion and inherent risk of neurological deterioration. Some of these cases may require a secondary surgical procedure at later stage. Some authors have advised perfect reduction to prevent late onset of neurological deterioration and spinal cord changes $[17,18]$.

The complications associated with neglected spinal injuries include pressure sores, back pain, urinary tract infections, gastrointestinal complaints, spasticity, contractures, depression and respiratory tract infections. Pressure sores was the major complication in these cases. The pressure sores in these patients developed mainly due to lack of compliance with rehabilitation program and neglect at home. In some cases, the pressure sores had progressed to osteomyelitis of the underlying bone. Few patients had experienced pain in the back, which could have been due to extensive paraspinal muscular striping and dissection during the surgery leading to muscular denervation and atrophy [19].

Limitations of the study were its retrospective design, lack of control group and high dropout rate. Various other parameters like site and extent of injury can affect the final functional outcome. Larger prospective studies are required to arrive at definite conclusion in the management of such injuries. However, this study emphases the point at which surgical intervention should be carried out even in delayed or missed cases, as there are significant chances of improvement in ASIA grades and future quality of life. The study shows importance of providing adequate infrastructure, trained manpower and awareness about the seriousness of the spine injury at primary health care level. This study also provides material for health care givers to pursue their cases for neglected spine rehabilitation services in this part of the world. 


\section{Conclusions}

The management of neglected traumatic spinal injuries is challenging with respect to intraoperative difficulties encountered, higher incidence of intraoperative and postoperative complications, inferior end results, requirement for additional surgical procedures and subsequent rehabilitation. This study highlights the difference in the reasons for neglected spinal injuries in developed and developing nations. Inadequate treatment at the initial contact due to lack of adequate infrastructure and trained manpower, non-availability or complete absence of health care facilities, opting for other treatment modalities in preference to modern medicine, poor rehabilitation subsequent to initial treatment, ignorance among patients and physicians about the seriousness of the spinal injuries were the chief reasons for neglected spine injuries in third world countries in comparison to overlooked or missed diagnosis in developed nations. The study may help in development of specific management strategies and interventions required in the management of neglected traumatic spinal injuries.

\section{Conflict of Interest}

No potential conflict of interest relevant to this article was reported.

\section{Acknowledgments}

The authors thank Mr. Baltej Singh, statistician, Department of Community Medicine, Guru Gobind Singh Medical College, Faridkot, India for conducting statistical analysis for the study.

\section{References}

1. Chhabra HS, Arora M. Demographic profile of traumatic spinal cord injuries admitted at Indian Spinal Injuries Centre with special emphasis on mode of injury: a retrospective study. Spinal Cord 2012;50:74554.

2. Jain AK, Dhammi IK, Singh AP, Mishra P. Neglected traumatic dislocation of the subaxial cervical spine. J Bone Joint Surg Br 2010;92:246-9.

3. Sengupta DK. Neglected spinal injuries. Clin Orthop Relat Res 2005;(431):93-103.

4. Catz A, Itzkovich M. Spinal Cord Independence
Measure: comprehensive ability rating scale for the spinal cord lesion patient. J Rehabil Res Dev 2007; 44:65-8.

5. Liu P, Zhao J, Liu F, Liu M, Fan W. A novel operative approach for the treatment of old distractive flexion injuries of subaxial cervical spine. Spine (Phila Pa 1976) 2008;33:1459-64.

6. Goni V, Gopinathan NR, Krishnan V, Kumar R, Kumar A. Management of neglected cervical spine dislocation: a study of six cases. Chin J Traumatol 2013;16:212-5.

7. Poonnoose PM, Ravichandran G, McClelland MR. Missed and mismanaged injuries of the spinal cord. J Trauma 2002;53:314-20.

8. Meldon SW, Moettus LN. Thoracolumbar spine fractures: clinical presentation and the effect of altered sensorium and major injury. J Trauma 1995;39:11104.

9. Stiell IG, Clement CM, McKnight RD, et al. The Canadian C-spine rule versus the NEXUS low-risk criteria in patients with trauma. N Engl J Med 2003; 349:2510-8.

10. Hendey GW, Wolfson AB, Mower WR, Hoffman JR; National Emergency X-Radiography Utilization Study Group. Spinal cord injury without radiographic abnormality: results of the National Emergency $\mathrm{X}$-Radiography Utilization Study in blunt cervical trauma. J Trauma 2002;53:1-4.

11. Krueger MA, Green DA, Hoyt D, Garfin SR. Overlooked spine injuries associated with lumbar transverse process fractures. Clin Orthop Relat Res 1996;(327):191-5.

12. Vaccaro AR, An HS, Lin S, Sun S, Balderston RA, Cotler JM. Noncontiguous injuries of the spine. J Spinal Disord 1992;5:320-9.

13. Albert TJ, Levine MJ, An HS, Cotler JM, Balderston RA. Concomitant noncontiguous thoracolumbar and sacral fractures. Spine (Phila Pa 1976) 1993;18:128591.

14. Reid DC, Henderson R, Saboe L, Miller JD. Etiology and clinical course of missed spine fractures. J Trauma 1987;27:980-6.

15. Bohlman HH. Acute fractures and dislocations of the cervical spine: an analysis of three hundred hospitalized patients and review of the literature. J Bone Joint Surg Am 1979;61:1119-42.

16. Jain AK, Dhammi IK, Jain S, Kumar J. Simultaneous- 
ly anterior decompression and posterior instrumentation by extrapleural retroperitoneal approach in thoracolumbar lesions. Indian J Orthop 2010;44:40916.

17. Malcolm BW, Bradford DS, Winter RB, Chou SN. Post-traumatic kyphosis: a review of forty-eight surgically treated patients. J Bone Joint Surg Am 1981;63:891-9.

18. Abel R, Gerner HJ, Smit C, Meiners T. Residual de- formity of the spinal canal in patients with traumatic paraplegia and secondary changes of the spinal cord. Spinal Cord 1999;37:14-9.

19. Fritzell P, Hagg O, Nordwall A; Swedish Lumbar Spine Study Group. Complications in lumbar fusion surgery for chronic low back pain: comparison of three surgical techniques used in a prospective randomized study: a report from the Swedish Lumbar Spine Study Group. Eur Spine J 2003;12:178-89. 